\title{
A compact, portable and low cost generic interrogation strain sensor system using an embedded VCSEL, detector and fibre Bragg grating
}

\author{
Graham C. B. Lee*a, Bram Van Hoe ${ }^{\mathrm{b}}$, Zhijun Yan ${ }^{\mathrm{a}}$, Oliver Maskery ${ }^{\mathrm{b}}$, Kate Sugden ${ }^{\mathrm{b}}$, David Webb ${ }^{\mathrm{a}}$, \\ Geert Van Steenberge ${ }^{c}$

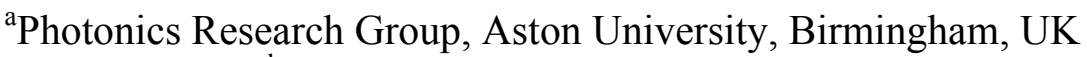 \\ ${ }^{\mathrm{b}}$ Astasense Ltd, Shrewsbury, UK \\ ${ }^{\mathrm{c}}$ Centre for Microsystems Technology (CMST), Elis Department, Ghent University - IMEC, \\ Belgium
}

\begin{abstract}
We present a compact, portable and low cost generic interrogation strain sensor system using a fibre Bragg grating configured in transmission mode with a vertical-cavity surface-emitting laser (VCSEL) light source and a GaAs photodetector embedded in a polymer skin. The photocurrent value is read and stored by a microcontroller. In addition, the photocurrent data is sent via Bluetooth to a computer or tablet device that can present the live data in a real time graph. With a matched grating and VCSEL, the system is able to automatically scan and lock the VCSEL to the most sensitive edge of the grating. Commercially available VCSEL and photodetector chips are thinned down to $20 \mu \mathrm{m}$ and integrated in an ultra-thin flexible optical foil using several thin film deposition steps. A dedicated micro mirror plug is fabricated to couple the driving optoelectronics to the fibre sensors. The resulting optoelectronic package can be embedded in a thin, planar sensing sheet and the host material for this sheet is a flexible and stretchable polymer. The result is a fully embedded fibre sensing system - a photonic skin. Further investigations are currently being carried out to determine the stability and robustness of the embedded optoelectronic components.
\end{abstract}

Keywords: Interrogation system, Vertical-cavity surface-emitting laser, fibre Bragg grating, flexible polymer skin, microcontroller, low cost, dynamic, flexible optoelectronic package.

\section{INTRODUCTION}

Fibre Bragg gratings (FBGs) are now widely used in sensing applications for temperature, strain and pressure. FBGs for sensing applications are readily available through commercial companies ${ }^{1,2}$. There are several different types of interrogation systems in the market; however, the majority are either bulky, expensive and or restricted in their functionality. In this paper we present a compact, portable and low cost generic interrogation strain sensor system using a FBG configured in transmission mode with a vertical-cavity surface-emitting laser (VCSEL) light source and a GaAs photodetector embedded in a polymer skin. The interrogation system is driven by a low-cost Atmel AVR microprocessor. The interrogation unit has two modes of operation - constant current mode and modulation mode. In constant current mode, the system automatically locks the VCSEL to the most sensitive part of the FBG and perturbations experienced by the sensor are displayed on a computer or Android tablet application. In modulation mode, the interrogation unit can dynamically modulate the light source up to a rate of $1 \mathrm{kHz}$. The dynamic modulation of the light source enables the system to detect very small perturbations on the sensor. Dynamic measurements capturing FBG spectral data at rates of $100 \mathrm{kHz}$ have previously been reported using a broadband source and a tuneable photodetector ${ }^{3}$. An ultra-thin package is developed to integrate the optoelectronics in thin flexible foils and a dedicated in-the-plane coupling technology is reported. The final sensing sheet consists of a FBG, embedded VCSEL and an embedded detector as described in Figure 1.

*leegcb@aston.ac.uk; phone +44 121204 3498; fax +44 1212043682; www.aston.ac.uk

Vertical-Cavity Surface-Emitting Lasers XVI, edited by Chun Lei, Kent D. Choquette, Proc. of SPIE Vol. 8276, 82760E · @ 2012 SPIE · CCC code: 0277-786X/12/\$18 · doi: 10.1117/12.907810 


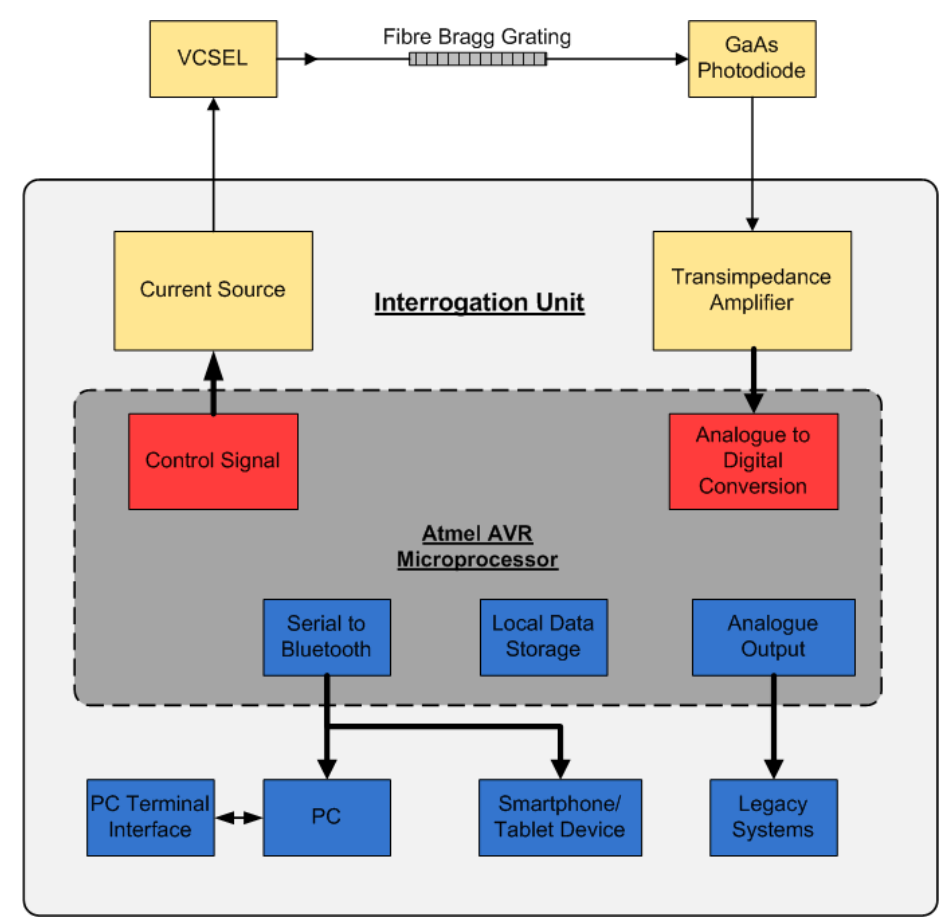

Figure 1. System Block Diagram

\section{ULTRA-THIN AND FLEXIBLE OPTOELECTRONIC COMPONENTS}

The FBG sensor used in this paper was fabricated using a UV holographic technique in Fibercore PS1250/1500 fibre. The physical size and characteristics of fibre sensors make them ideal for imbedding in a flexible polymer skin. The VCSEL and photodetector array components are bought commercially and are provided with a chip thickness of $125 \mu \mathrm{m}$. The two optoelectronic components are lapped and polished down to around $20 \mu \mathrm{m}$ while maintaining their electrical and optical characteristics. A new electrical back contact is applied by AuGeNiAu sputtering/evaporating. The optoelectronic components are subjected to a multistage packaging process described in Figure 2 to produce flexible and embedded components with a total thickness of only $40 \mu \mathrm{m}$. The optical characteristics of an embedded single-mode $850 \mathrm{~nm}$ VCSEL and photodetector are shown in Figure 3 and Figure 4 respectively.

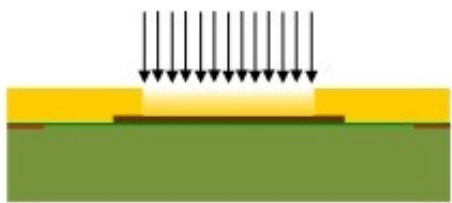

(b) Laser patterning cavity

(a) Polymer layer deposition on rigid glass carrier

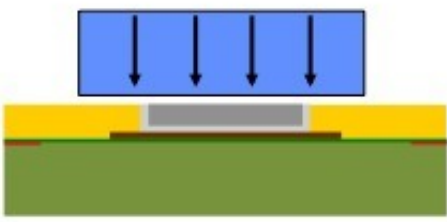

(d) Chip leveling inside cavity

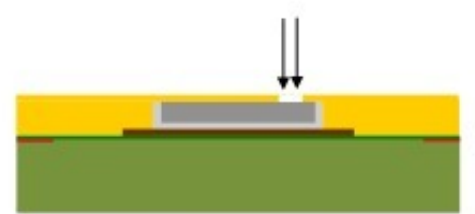

(e) Laser blind via drilling to chip contacts

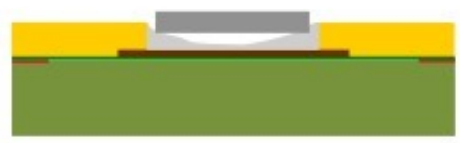

(c) Face-up chip placement and fixation

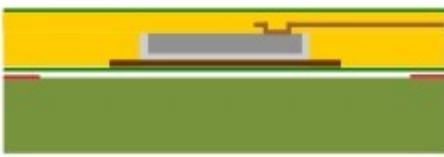

(f) Electrical contacting polymer layer deposition release from glass carrier

Figure 2. Thinning flexible embedded optoelectronic components. 
Figure 2. A polyimide layer and an epoxy SU-8 layer are spin coated consecutively on to a glass carrier (a). A copper island is deposited between the two layers to act as a heat sink. The SU-8 material on top of the heat sink is locally removed using $\mathrm{CO}_{2}$ and excimer laser ablation (b). A cavity is consequently created to allow the optoelectronic chip components to be positioned and mounted using an electrically conductive glue (c,d). A second spin-coated SU-8 layer covers the chip and contact pads are opened using excimer laser ablation (e). A galvanic fan-out is deposited and structured. Finally, another thin SU-8 layer and the top polyimide layer are spin coated (f). The flexible package is released from the processing carrier by laser cutting. More details on this embedding process can be found in reference ${ }^{4}$.
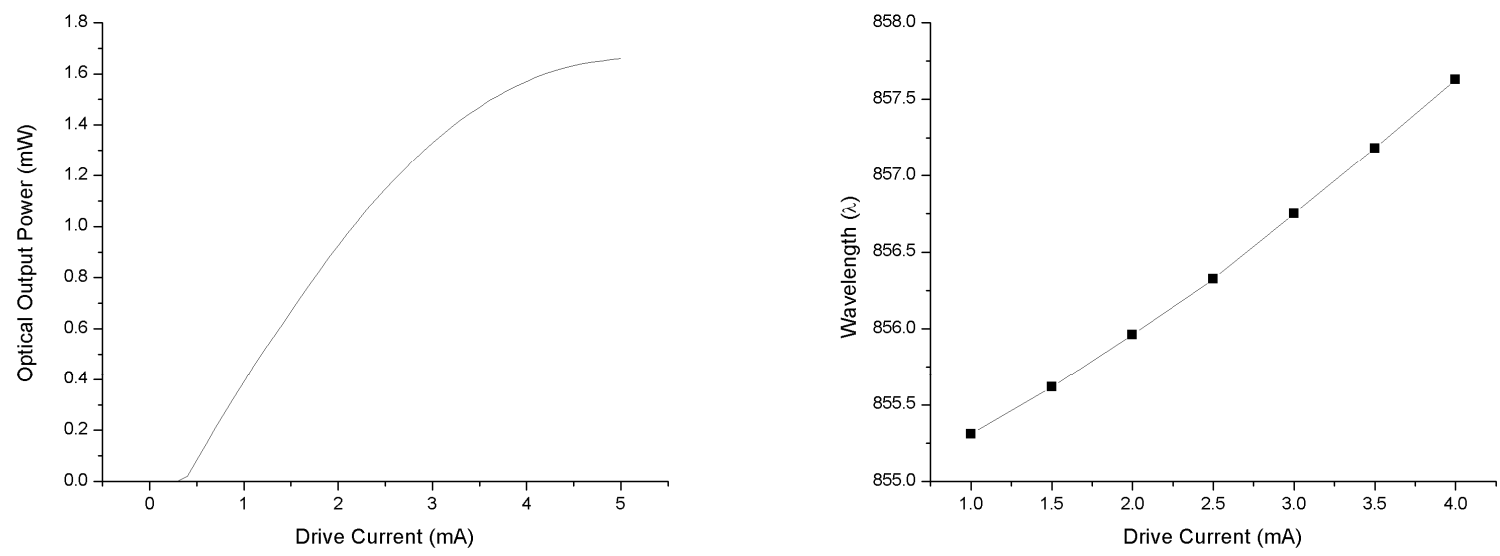

Figure 3. Embedded single-mode $850 \mathrm{~nm}$ VCSEL optical power and wavelength characteristics.

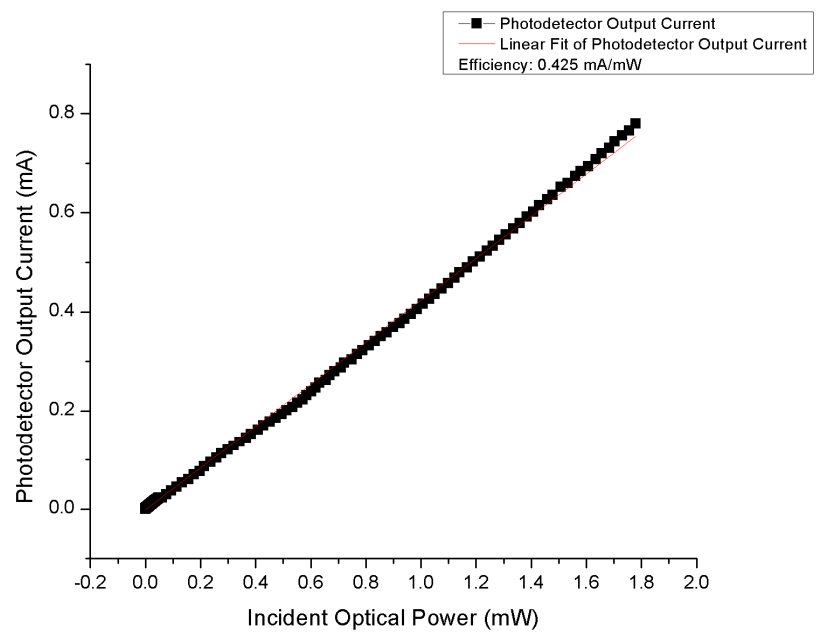

Figure 4. Photodetector characteristics

\subsection{Fibre Coupling}

A dedicated in-the-plane coupling scheme has been developed to allow light to be coupled in and out of the optoelectronic components when they are fully embedded in a flat sheet to form a polymer skin. The coupling is achieved through a PMMA plug (500 $\mu \mathrm{m}$ thickness) with a U- or V-groove to clamp the fibre as shown in Figure 5. Lapping and polishing techniques are employed to fabricate a $45^{\circ}$ mirror facet which is directly applied to the coupling fibre in the PMMA plug. This limits the optical path and consequently minimises the losses. The fibre is fixed with a special index-matched UV-glue and a reflecting layer is applied by chemically evaporating a $200 \mathrm{~nm}$ layer of gold. The 
coupling plug is mounted on top of the VCSEL package; therefore, the vertically emitted light is reflected on the gold surface through the coupling plug into the optical fibre.

The final device shown in Figure 6 features a smooth fibre facet of optical quality with an average surface roughness of $23 \mathrm{~nm}$ over a $50 \mu \mathrm{m} \times 50 \mu \mathrm{m}$ area. Coupling losses due to the fibre coupling plugs are found to be around 2 to $3 \mathrm{~dB}$ for VCSEL devices and around $1 \mathrm{~dB}$ for photodetectors.

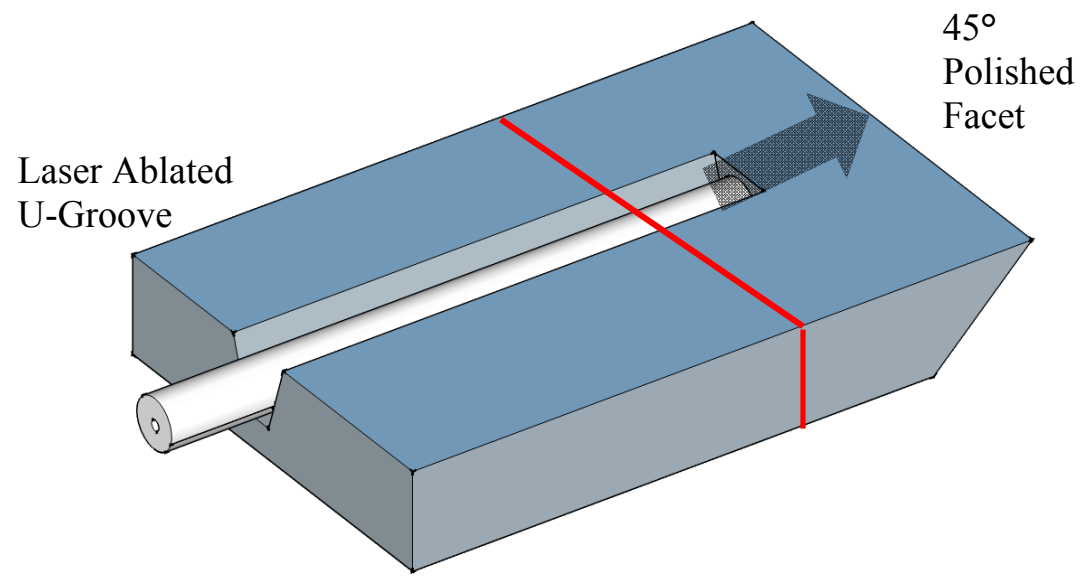

Figure 5. Schematic of fibre coupling scheme.
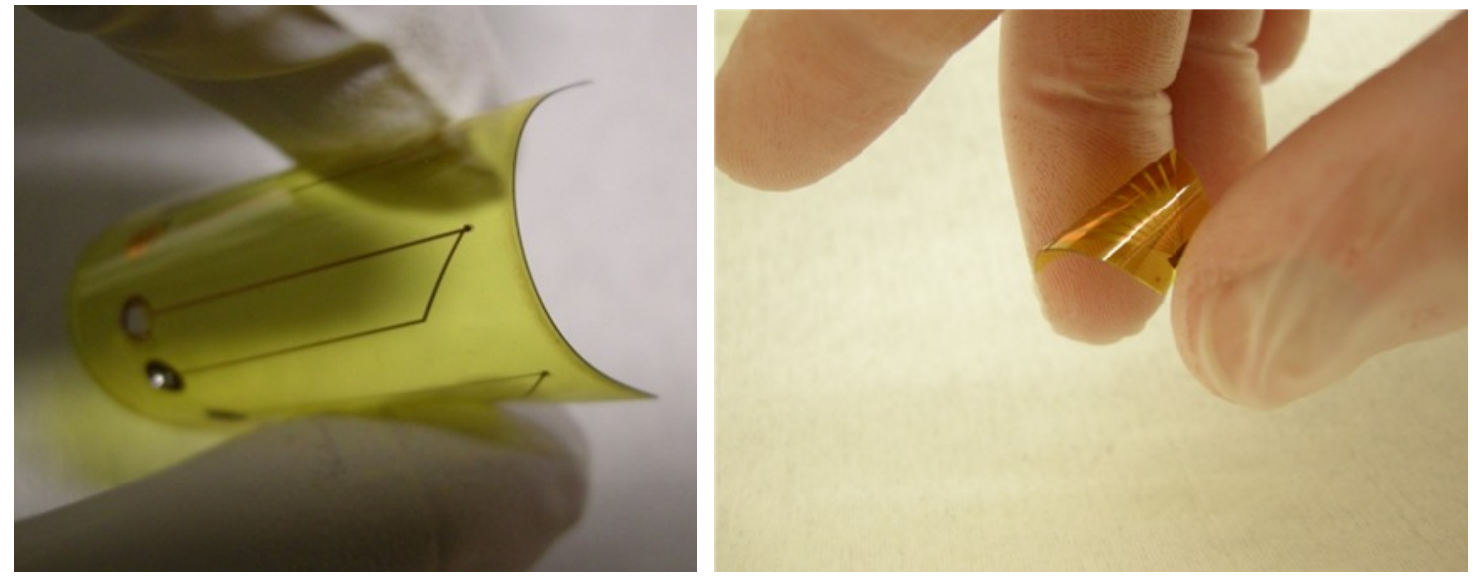

Figure 6. (Left) Embedded single-mode $850 \mathrm{~nm}$ VCSEL. (Right) Embedded photodetector array, total thickness $550 \mu \mathrm{m}$.

\section{INTERROGATION UNIT}

The interrogation unit measures $160 \mathrm{~mm} \times 116 \mathrm{~mm} \times 35 \mathrm{~mm}$ and weighs approximately 515 grams with a lithium ion battery. The unit is able to run continuously for up to 24 hours on one charge or it can operate from a power outlet. The compact, portable and low cost generic interrogation strain sensor system is built around an Atmel ATMEGA644 microcontroller. The low-power and high-performance Atmel ATMEGA644 microcontroller offers 64KB ISP flash memory, 2KB EEPROM, 4KB SRAM, 8 or 10-bit analogue to digital convertor, 2 USARTs and 32 general purpose input and output lines. This makes the Atmel ATMEGA microcontroller family both low cost and sufficiently powerful enough to drive all the necessary components to form a flexible and full-featured interrogation system.

The microcontroller interfaces with a Wavelength Electronics LDD P series current source in an integrated package. The current source is able to drive a light source up to $120 \mathrm{~mA}$. The current source can operate in constant current mode or it can be driven by a modulation signal generated by the microcontroller. The photodetector in the interrogation system is 
connected through a low noise transimpedance amplifier with a variable gain and into one of the analogue to digital convertor ports of the microcontroller. The variable gain in the transimpedance amplifier allows the user to tune the sensitivity of the interrogation system to suit the strength of the light source, grating and photodiode.
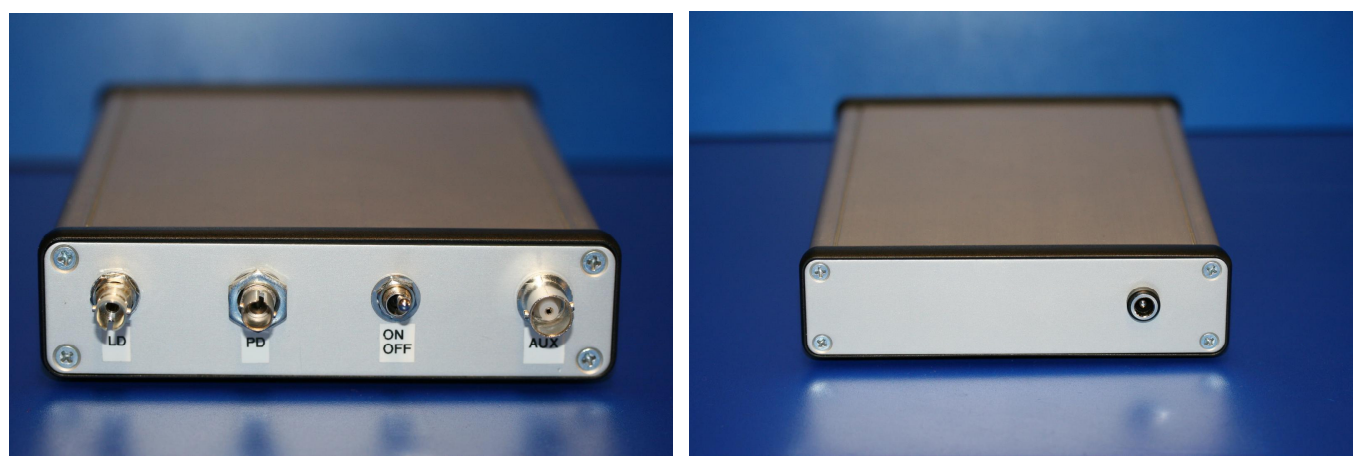

Figure 7. (Left) Front panel. (Right) Rear panel, power input.

The interrogation unit has two modes of operation - constant current mode and modulation mode. In constant current mode, the unit systematically modulates the light source to determine the position of the grating and the most sensitive position on the grating. Once the most sensitive point of the grating has been found, the interrogation unit automatically sets the corresponding light source drive current. At a set drive current, the interrogation unit samples the photodetector to monitor for any perturbations on the grating. The microcontroller samples the photodetector at $5 \mathrm{kHz}$; averaging of several data readings can be carried out directly on the microcontroller.

In modulation mode, the user is able to select a type of modulation signal such as a saw tooth signal to dynamically drive the current source at a frequency up to $1 \mathrm{kHz}$. Modulating the VCSEL provides a dynamic reconstruction of the grating spectrum; this is schematically shown in Figure 8.

(a)

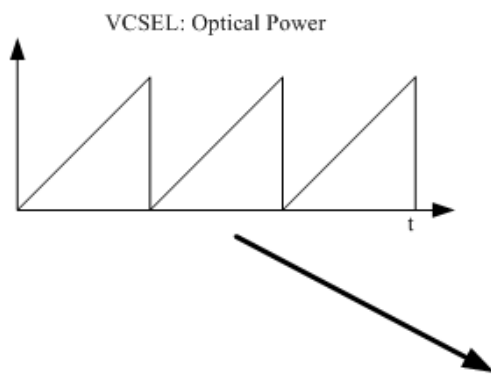

(b)
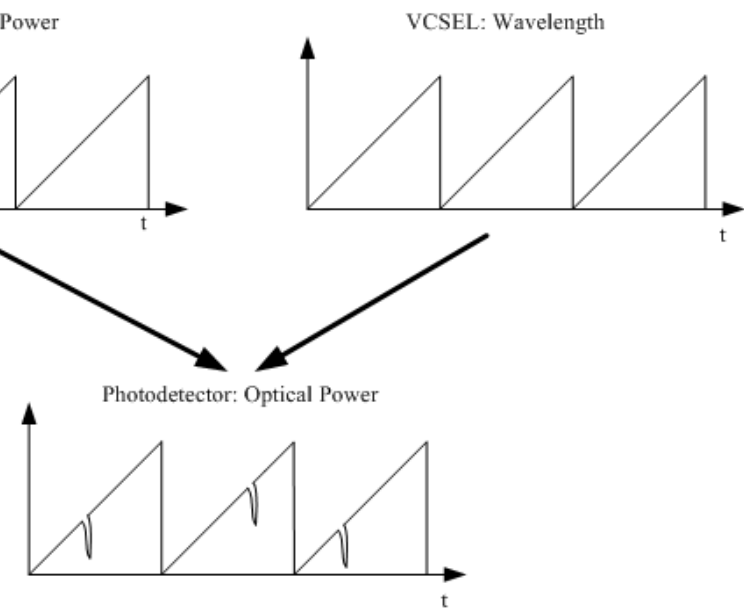

Figure 8. Modulation mode - interrogation scheme.

\subsection{Read out}

The interrogation unit features several output lines that are fully configurable by the user. The serial RS232 connection is the primary interface for extracting data from the system. The user is able to setup and view the real time data from the system through a computer terminal interface connected over RS232 or over Bluetooth by employing a serial to Bluetooth adapter. An Android tablet application shown in Figure 9 forms the main method for configuring, viewing and 
storing the live data streamed from the interrogation unit. The Android tablet can automatically establish a Bluetooth connection to the interrogation unit. Once connected, a measurement session begins where a real time graph is immediately displayed showing incident light on the photodetector against a data reading identifier. Data received by the tablet device are logged and stored which can later be retrieved for analysis using the multi-touch display.

The auxiliary port provides a simple and fast method for viewing the real time data when data storage is not required. The auxiliary port is also suitable for connecting the interrogation system to legacy equipment. In addition, the $2 \mathrm{~KB}$ of EEPROM memory built into the microcontroller provides sufficient memory to store at least 500 readings if required.

A large amount of data is collected and analysed when the interrogation unit is set to modulation mode. This is due to the high sampling rate required to reconstruct the grating spectrum. When in modulation mode, data is best viewed on an oscilloscope using the auxiliary port to avoid reaching the limit of the processing capacity of the microcontroller; this is highlighted in Figure 11 and Video 1.

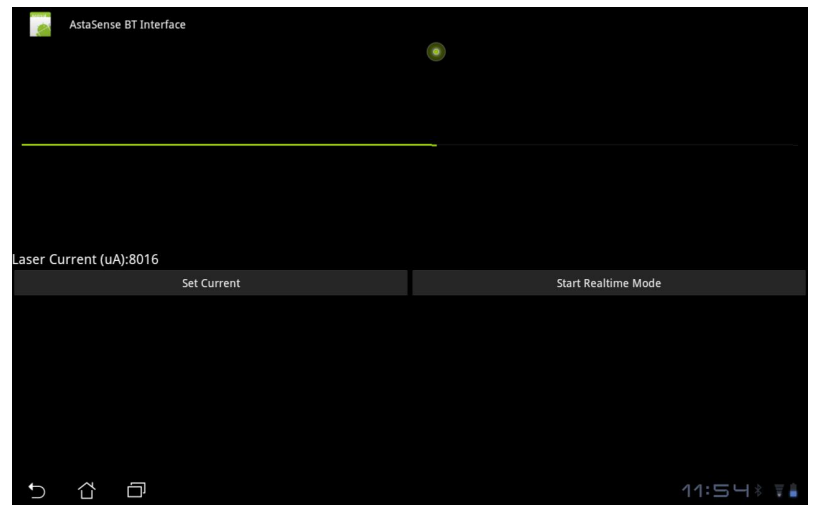

Figure 9. Android application graphical user interface.

\section{EMBEDDED SYSTEM DEMONSTRATION}

The embedded optoelectronic components described in section 2 and the interrogation unit described in section 3 are used in this demonstration. The FBG is attached to a beam that is fixed at one end, while the other end can be manually tapped and made to resonate. The interrogation unit is configured in constant current mode to show the whole system working from a tablet device. On start-up, the interrogation unit sweeps the VCSEL drive current from $0.1 \mathrm{~mA}$ to $4 \mathrm{~mA}$ with a step size of $0.01 \mathrm{~mA}$. Since there is a broadly linear relationship between the electrical current and VCSEL wavelength the VCSEL is swept from $855.877 \mathrm{~nm}$ to $857.660 \mathrm{~nm}$. As the light source is swept, the microcontroller samples the photodetector and establishes the most sensitive part of the grating. Once the most sensitive part of the grating is found the microcontroller automatically sets the corresponding VCSEL current. After the VCSEL drive current has been successfully set, the beam was manually tapped and made to resonate. The photodetector was sampled at $5 \mathrm{kHz}$, every 50 readings were averaged and plotted on the Android tablet in real time as shown in Figure 10.

Figure 11 shows the interrogation unit in modulation mode using the same optoelectronics components. A 50:50 coupler was introduced to the optical setup to observe the FBG in reflection. The coupler was added to the setup so that the grating can easily be observed with the resolution that can be easily be achieved with the interrogation unit and tablet device when in modulation mode. Video 1 shows an example of the dynamic read out using an oscilloscope with the optics setup in transmission mode. The reconstructed grating spectrum in Figure 11 is not as clearly resolved when compared to the setup in Video 1, this is due to the limitation of the processing capacity of the microprocessor. 


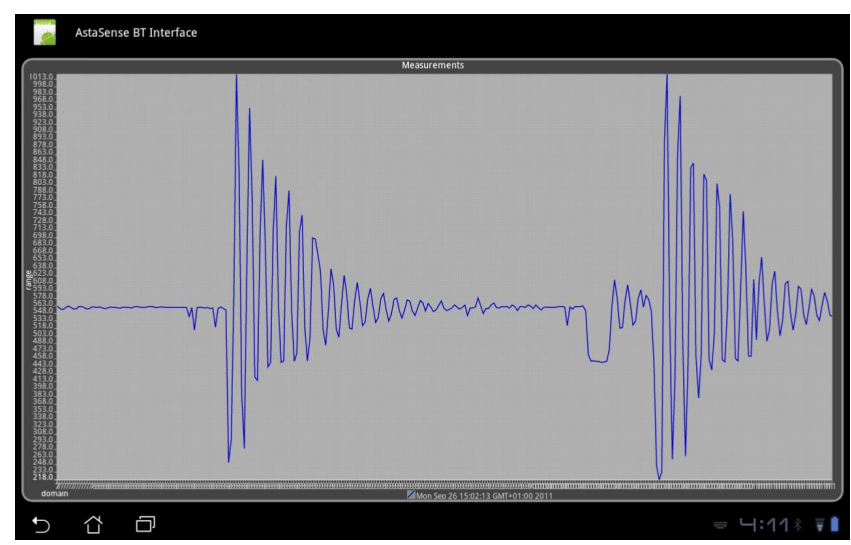

Figure 10. Photodetector current plotted against data reading identifier. Waveform shows the response of a fibre Bragg grating that has been attached to a beam fixed at one end and made to resonate.
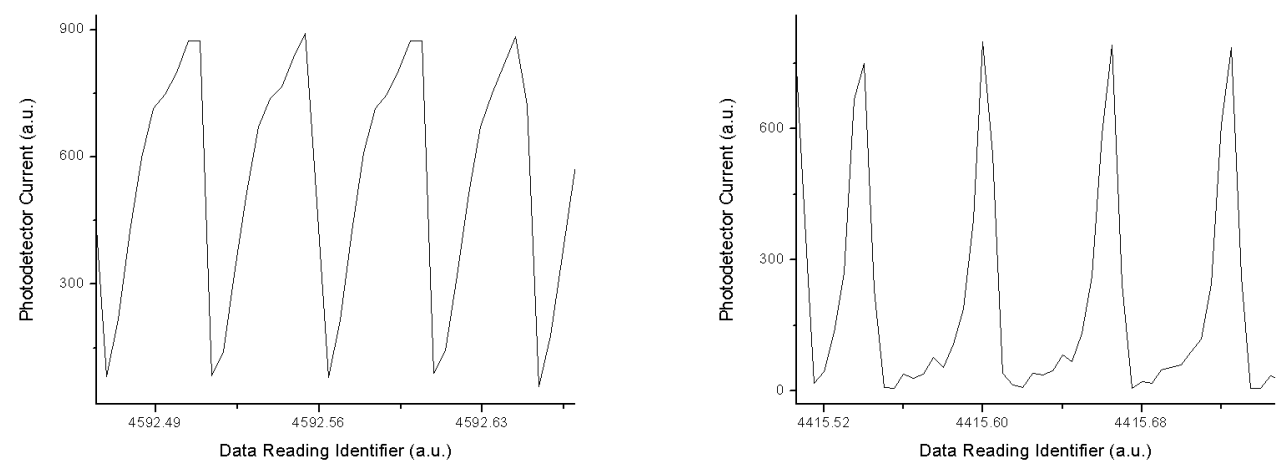

Figure 11. (Left) $6 \mathrm{~Hz}$ saw tooth modulation signal generated by interrogation unit with no grating. (Right) $6 \mathrm{~Hz}$ saw tooth modulation signal generated by interrogation unit with FBG in reflection.

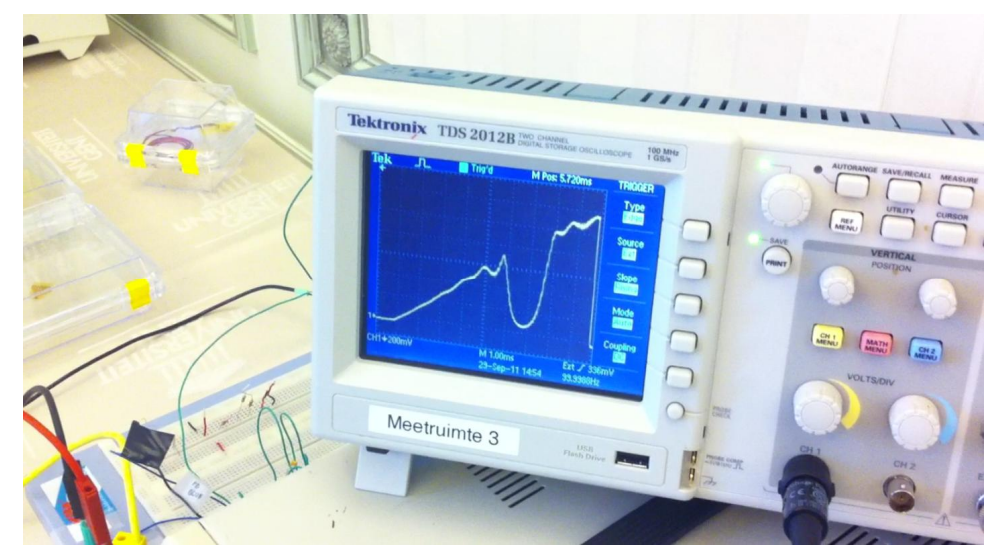

Video 1. Modulation mode - dynamic read out demonstration with grating in transmission. $\underline{\text { http://dx.doi.org/10.1117/12.907810.1 }}$ 


\section{CONLUSION}

We have developed a compact, portable and low cost generic strain sensor system. A functional system has been demonstrated with an embedded VCSEL, photodetector and fibre Bragg grating in transmission. We are currently refining the embedding process to form a fully embedded system. In addition, further investigations are being carried out to determine the stability and robustness of the embedded optoelectronic components.

\section{ACKNOWLEDGMENTS}

This work is partially conducted in the framework of the project PHOSFOS 5 , Photonic Skins for Optical Sensing (funded within the EU-FP7 program). G. Lee thanks the Engineering and Physical Sciences Research Council (EPSRC) for funding his Ph.D.

\section{REFERENCES}

[1] http://www.fos-s.be

[2] http://www.tft-fos.com

[3] T. Vella, S. Chadderdon, R. Selfridge, S. Schultz, S. Webb, C. Park, K. Peters and M. Zikry, "Full-spectrum interrogation of fiber Bragg gratings at $100 \mathrm{kHz}$ for detection of impact loading", Meas. Sci. Technol. 21, 094009 (2010).

[4] E. Bosman, J. Missinne, B. Van Hoe, G. Van Steenberge, S. Kalathimekkad, J. Van Erps, I. Milenkov, K. Panajotov, T. Van Gijseghem, P. Dubruel, H. Thienpont and P. Van Daele, "Ultrathin Optoelectronic Device Packaging in Flexible Carriers", IEEE Journal of Selected Topics in Quantumelectronics, 17(3), 617-628 (2011).

[5] http://www.phosfos.eu 